\title{
NOTIZEN
}

\section{Reduktion von Halogenen im Ultraschallfeld}

Wiktor Wawrzyczek und Zdzislaw Bieliński

Institut für Allgemeine Chemie der Landwirtschaftlichen Hochschule, Olsztyn, Polen

(Z. Naturforschg. 20 b, 595-596 [1965] ; eingeg. am 18. Januar 1965)

Soweit bisher Arbeiten über das aktive Einwirken von Ultraschallwellen auf chemische Prozesse vorliegen, handelt es sich ausschließlich um Oxydations-Reaktionen von Halogeniden in wäßrigen Lösungen ${ }^{1-5}$. Unter den Reaktionen

$$
\mathrm{X}^{\ominus}-\mathrm{e}=\mathrm{X}
$$

ist am gründlichsten die Oxydations-Reaktion der wäßrigen KJ-Lösungen bearbeitet.

In dieser Arbeit wird versucht, den umgekehrten Prozeß im Ultraschallfeld

$$
\mathrm{X}+\mathrm{e}=\mathrm{X}^{\ominus}
$$

darzustellen.

Hierzu wurden stets $50 \mathrm{ml}$ wäßriger Chlor-, Bromoder Jodlösungen verschiedener Konzentration eingesetzt. Zur Beschallung diente ein Ultraschall-Quarzgenerator, elektrische Leistung 2-7 W/ $\mathrm{cm}^{2}$, Frequenz $1000 \mathrm{kHz}$, Beschallungsdauer 3, 5, 10 und 15 Minuten. Das Reaktionsgefäß mit sehr dünnem Glasboden, dessen Durchmesser kleiner ist als der Durchmesser der schwingenden Quarzplatte, wurde laufend mit Wasser gekühlt. Die Temperatur der beschallten Flüssigkeit betrug etwa $21{ }^{\circ} \mathrm{C}$. Die Beschallung erfolgte bei Atmosphärendruck entweder in Luftatmosphäre oder in Argon. Im letzteren Fall ließ man durch die Lösung, der zuvor die Luft entzogen wurde, etwa \pm 50 Bläschen Argon pro Min. hindurchströmen. Das Reaktionsgefäß befand sich stets in derselben Entfernung (3 mm) von der Quarzplatte. Unmittelbar nach der Beschallung wurden nach der M o h r schen Methode die Konzentrationen der $\mathrm{Cl}^{\ominus}$. und $\mathrm{Br}^{\ominus}$.Ionen bestimmt; die Konzentration der $\mathrm{J}^{\ominus}$-Ionen wurde aus dem Mengenunterschied von Jod vor und nach der Beschallung ermittelt und zwar durch Titrieren von $20 \mathrm{ml} 0,01-n$. $\mathrm{Na}_{2} \mathrm{~S}_{2} \mathrm{O}_{3}$-Lösung in Gegenwart von Stärke. Die erhaltenen Ergebnisse wurden denen aus nicht beschallten Lösungen gegenübergestellt und verglichen.

\section{Ergebnisse und Diskussion}

Unter der Einwirkung von Ultraschallwellen zerfällt Wasser in $\mathrm{H}$ - und $\mathrm{OH}$-Radikale, die in sonochemischen Prozessen von entscheidender Bedeutung sind. In An-

${ }^{1}$ H. H. Rust, Angew. Chem. 64, 162 [1952].

2 O. Lindström, J. acoust. Soc. America 27, 654 [1955].

3 H. Gueguen, Bull. Soc. chim. France 2, 202 [1959] ; 7, 1254 [1959]. wesenheit von Sauerstoff bilden sich $\mathrm{HO}_{2}$-Radikale mit schwacher Oxydationswirkung. Infolge von Ionisation $\left(\mathrm{HO}_{2}=\mathrm{H}^{\oplus}+\mathrm{O}_{2}{ }^{\ominus}\right)$ haben $\mathrm{O}_{2}{ }^{\ominus}$-Ione eine größere Reduktionswirkung. Die entstandenen Radikale reagieren entweder gegenseitig miteinander, indem sie eine ganze Reihe von Nebenprodukten liefern, oder mit in Wasser gelösten Substanzen. Je nach dem gegenseitigen Mengenverhältnis dieser Radikale und dem OxydoredoxPotential der aufgelösten Substanz lassen sich während der Beschallung Reduktions- beziehungsweise Oxydationsprozesse beobachten.

1. Sofern die elektrische Leistung des Ultraschallfeldes ansteigt, nimmt die Reaktionsausbeute

$$
\mathrm{X}+\mathrm{e}=\mathrm{X}^{\ominus}
$$

bei sämtlichen Konzentrationen der untersuchten Halogene ab. Sobald ferner die Stärke der Ultraschallwelle unter den Schwellenwert der Kavitation sinkt, läßt sich kein sonochemischer Prozeß beobachten. Es existiert folglich eine optimale elektrische Leistung, welche die größte Menge von Kavitationsbläschen hervorzurufen vermag. Weitere Steigerung der elektrischen Leistung hat eine Verringerung der Bläschenbildung zur Folge. Der Grund hierfür liegt wahrscheinlich in der Entziehung des Gases bis zu einem solchen Grad, daß der Gasrest nicht mehr imstande ist, Kavitationsbläschen zu bilden, die für das Zustandekommen des sonochemischen Effektes verantwortlich sind.

2. Die Reduktionsreaktion der Halogene im Ultraschallfeld kann man mittels der Gleichungen

$$
\begin{gathered}
\mathrm{X}_{2}=\mathrm{X}+\mathrm{X} \\
\mathrm{X}+\mathrm{H}=\mathrm{H}^{\oplus}+\mathrm{X}^{\ominus}
\end{gathered}
$$

und in Gegenwart von Sauerstoff mittels der Gleichung

$$
\mathrm{X}+\mathrm{O}_{2}{ }^{\ominus}=\mathrm{X}^{\ominus}+\mathrm{O}_{2}
$$

darstellen.

Wie bereits a priori auf Grund der Kenntnis der Oxydationspotential-Werte von Halogenen vorauszusehen war, verläuft der Reduktionsprozeß mit dem Anwachsen des Atomgewichtes bei den Halogenen schwächer. Daraus geht hervor, daß die $\mathrm{Cl}^{\ominus}$-Ionen schlechtere Auffänger von $\mathrm{OH}$-Radikalen sind als $\mathrm{Br}^{\ominus}$. und $\mathrm{J}^{\ominus}$-Ionen, die eine entgegengesetzte Reaktion hervorrufen.

$$
\mathrm{X}^{\ominus}+\mathrm{OH}=\mathrm{X}+\mathrm{OH}^{\ominus} \text {. }
$$

3. Die ausgeschiedene $\mathrm{X}^{\ominus}$-Ionmenge wächst zugleich mit der Konzentration der entsprechenden Halogenlösung an. Je größer die Konzentration, desto deut-

4 W. Wawrzyczek u. D. Tylzanowska, Naturwissenschaften 49, 58 [1962].

5 W. W awrzyczek u. A. Przybylski, Z. anorg. allg. Chem. 314, 285 [1962]. 
licher macht sich der Einfluß des Gases geltend; je schwächer die Konzentration, desto unscheinbarer ist die Rolle des aufgelösten Gases. Die Effekte der sonochemischen Reaktion ähneln sich in diesen Fällen sowohl in Anwesenheit von Luft als auch von Argon. Chlor desoxydiert in Gegenwart von Luft am besten, Jod dagegen am schlechtesten, da es unter den Halogenen zu den schwächsten Oxydanten zählt. In Gegenwart von Argon beobachtet man die umgekehrte Erscheinung. Nach Virtanen und Ellfolk ${ }^{6}$ bilden sich während der Beschallung der Lösung im Beisein von Luft verschiedene Produkte der Stickstoffoxydation. In unserem Fall verhindert die Reaktion

$$
\mathrm{NO}_{2}{ }^{\ominus}+\mathrm{Cl}=\mathrm{NO}_{2}+\mathrm{Cl}^{\ominus}
$$

deutlich die Reaktion

$$
\mathrm{Cl}^{\ominus}+\mathrm{OH}=\mathrm{Cl}+\mathrm{OH}^{\ominus} .
$$

Brom reduziert besser als Jod, jedoch schlechter als Chlor, was seinem Oxydationspotential-Wert entspricht. Argonatmosphäre begünstigt die Reaktion

$$
\mathrm{J}+\mathrm{H}=\mathrm{J}^{\ominus}+\mathrm{H}^{\oplus},
$$

da in Gegenwart von Argon eine Rekombination von $\mathrm{OH}$-Radikalen in $\mathrm{H}_{2} \mathrm{O}_{2}$-Moleküle nach der Gleichung

$$
\mathrm{OH}+\mathrm{OH}+\mathrm{Ar}=\mathrm{H}_{2} \mathrm{O}_{2}+\mathrm{Ar}
$$

vor sich geht. $\mathrm{H}_{2} \mathrm{O}_{2}$-Moleküle im entsprechenden Verhältnis $[\mathrm{J}]:\left[\mathrm{J}^{\ominus}\right]$ können zusätzlich eine bestimmte Menge von Jod gemäß nachfolgender Gleichung reduzieren:

oder

$$
2 \mathrm{~J}+\mathrm{H}_{2} \mathrm{O}_{2}=2 \mathrm{H}^{\oplus}+2 \mathrm{~J}^{\ominus}+\mathrm{O}_{2}
$$

$$
\mathrm{J}_{2}+\mathrm{H}_{2} \mathrm{O}_{2}=2 \mathrm{H}^{\oplus}+2 \mathrm{~J}^{\ominus}+\mathrm{O}_{2} .
$$

6 A. I. Virtanen u. N. Ellfolk, Acta chem. scand. 4, 93 [1950].

\section{NMR-Untersuchungen über Selen- und Tellurwasserstoffe}

\author{
H. Schmidbaur und W. Siebert \\ Institut für Anorganische Chemie der Universität \\ Marburg/Lahn \\ (Z. Naturforschg. 20 b, 596-597 [1965] ; eingeg. am 7. Januar 1965)
}

Zur Klärung vieler Probleme der Chemie der Polyschwefelwasserstoffe ist die NMR-Spektroskopie besonders geeignet, da sie eine einfache qualitative und quantitative Registrierung der einzelnen Homologen $\mathrm{H}_{2} \mathrm{~S}_{\mathrm{x}}$ ermöglicht ${ }^{1,2}$. Wir haben jetzt versucht, mit dieser Methode auch die bisher unsichere Existenz der Polyselen- und -tellurwasserstoffe nachzuweisen. Dies ist uns jedoch trotz mehrfacher Variation der Versuchsbedingungen nicht gelungen. Alle Experimente zur Freisetzung von $\mathrm{H}_{2} \mathrm{Se}_{\mathrm{x}}$ und $\mathrm{H}_{2} \mathrm{Te}_{\mathrm{x}}$ aus Alkalipolyseleniden bzw. -telluriden mit wäßrigen Säuren wechselnder Kon-

1 H. Schmidbaur u. W. Siebert, Chem. Ber. 97, 2090 [1964].

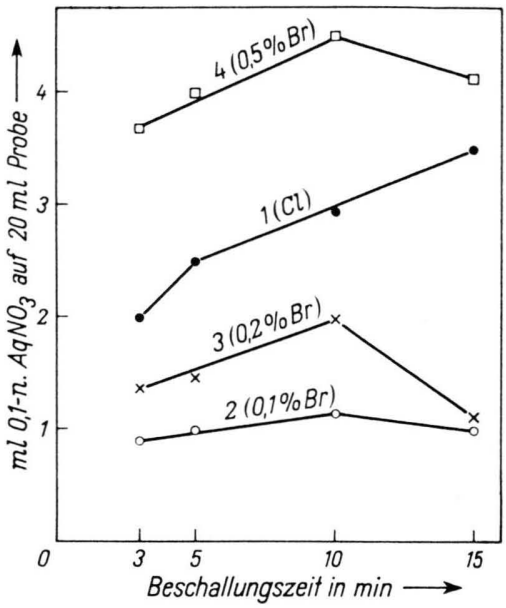

Abb. 1. Beziehung zwischen Beschallungszeit und Entstehung von $\mathrm{Cl}^{\ominus}$., $\mathrm{Br}^{\ominus}$ - und $\mathrm{J}^{\ominus}$.Ionen.

4. In gesättigten wäßrigen Chlorlösungen in einer Temperatur von $21{ }^{\circ} \mathrm{C}$ wächst die Menge der ausgeschiedenen $\mathrm{Cl}^{\ominus}$-Ionen fast proportional zur Beschal lungszeit (3, 5, 10 und 15 Min.) an (s. Abb. 1, Kurve 1).

In Lösungen, die $0,1,0,2$ und $0,5 \% \mathrm{Br}$ enthalten, beobachtet man bis zu einer Beschallungsdauer von 10 Min. ein ständiges Anwachsen der Konzentration von $\mathrm{Br}^{\ominus}$. Bei länger währender Beschallung tritt Reduktionsschwund und sogar teilweise Verringern der $\mathrm{Br}^{\ominus}$-Menge ein (Abb. 1, Kurve 2, 3 und 4).

Lösungen, die $0,24 \% \mathrm{~J}$ enthalten, liefern $\mathrm{J}^{\ominus}$-Mengen, die in Proportion zur Beschallungszeit stehen. Stärker verdünnte Lösungen $(0,0008 \%)$ verhalten sich ähnlich wie Bromlösungen.

zentration bei -25 bis $+25^{\circ}$ lieferten nur $\mathrm{H}_{2} \mathrm{Se}$ und Selen bzw. $\mathrm{H}_{2} \mathrm{Te}$ und Tellur.

\begin{tabular}{|c|c|c|c|c|}
\hline & $\delta_{\mathrm{H}}$ & $\Delta \delta_{\mathrm{H}}$ & $J(\mathrm{H}-\mathrm{X}-\mathrm{D})$ & $J(\mathrm{H}-\mathrm{X}-\mathrm{H})$ \\
\hline $\mathrm{HSeH}$ & $+63,7$ & 10 & - & 13,7 \\
\hline HSeD & $+65,8$ & & $\pm 2,10$ & - \\
\hline HTeH & $+323,5$ & +210 & - & 13,7 \\
\hline HTeD & $+325,6$ & & $\pm 2,10$ & - \\
\hline
\end{tabular}

Bei diesen Untersuchungen haben wir aber die bislang unbekannten Werte der Chemischen Verschiebung des $\mathrm{H}_{2} \mathrm{Se}$ und $\mathrm{H}_{2}$ Te sowie ihrer monodeuterierten Derivate $\mathrm{HSeD}$ und $\mathrm{HTeD}$ in Schwefelkohlenstoff bestimmt (Tab. 1).

Tab. 1. Chemische Verschiebungen $\delta_{\mathrm{H}}, \mathrm{H} / \mathrm{D}$-Isotopeneffekt $\Delta \delta_{\mathrm{H}}$, und Kopplungskonstanten $J(\mathrm{HXH})$ und $J(\mathrm{HXD})$ bei schweren Chalkogenwasserstoffen. [Alle Daten in $\mathrm{Hz}$ bei $60 \mathrm{MHz}$. Gemessen an (bei $0^{\circ}$ ) gesättigten Lösungen in $\mathrm{CS}_{2}$ bei $35^{\circ}$ unter Eigendruck. TMS als int. Std., positive $\delta$-Werte für zunehmende Feldstärken. Fehlergrenzen $\pm 0,1 \mathrm{~Hz}$ für $J$ und $\Delta \delta, \pm 2,0$ für $\left.\delta_{\mathrm{H}} . \mathrm{X}=\mathrm{Se}, \mathrm{Te}.\right]$

2 H. Schmidbaur, M. Schmidt u. W. Siebert, Chem. Ber. 97, 3374 [1964]. 